\title{
Recombinant adeno-associated viruses (rAAV2) facilitate the intraperitoneal gene delivery to cancer cells
}

\author{
MACIEJ MALECKI ${ }^{1,2}$, ROBERT PROCZKA ${ }^{3}$, JOANNA CHOROSTOWSKA-WYNIMKO ${ }^{5}$, \\ PAWEŁ SWOBODA ${ }^{1}$, ANNA DELBANI ${ }^{4}$ and JAN PACHECKA ${ }^{2}$ \\ ${ }^{1}$ Department of Cell Biology, Centre of Oncology, Maria Sklodowska-Curie Memorial Institute; \\ ${ }^{2}$ Department of Biochemistry and Clinical Chemistry, ${ }^{3}$ Second Department of General, Vascular and \\ Oncological Surgery and ${ }^{4}$ Department of Pharmacy, Medical University of Warsaw; ${ }^{5}$ Laboratory of \\ Molecular Diagnostics, National Institute of Tuberculosis and Lung Diseases, Warsaw, Poland
}

Received February 12, 2009; Accepted July 29, 2009

DOI: 10.3892/ol_00000032

\begin{abstract}
Peritoneal dissemination of cancer cells is characteristic of advanced stages of ovarian, breast and lung cancers, and is associated with poor patient survival. The presence of cancer cells in effusions complicates treatment protocols, while cell eradication is seriously limited. One of the novel options available is cancer gene therapy with recombinant adeno-associated viruses. This combination represents the most promising gene delivery vehicles to neoplasmatic cells within serosal cavities due to their unique properties that include the ability to infect proliferating cells of broad host range, as well as the potential of long-term expression. Recombinant infectious adeno-associated virus serotype 2 particles (rAAV2) were produced in a helper-free system using an AAV-293 packaging cell line, and quantitatively analyzed by real-time PCR. Balb/c mice intraperitoneally pre-injected with L1 cancer cells were treated with different doses of rAAV2. Subsequently, the mice were sacrificed and intraperitoneal effusions were analyzed for $\mathrm{rAAV}$ presence and $\mathrm{rAAV} / \beta$-galactosidase ( $\mathrm{LacZ}$ ) vector efficiency in order to infect cancer cells within the peritoneal cavity. We reported an efficient infection of L1 cancer cells disseminated into the peritoneal cavity by rAAV2. The expression of reporter genes (GFP and $L a c Z$ ) attributable to the rAAV cell uptake was closely dependent on an applied multiplicity of infection ratio (MOI). The highest infection efficiency was observed at a MOI of 50 and 200. Our study confirmed the ability of adeno-associated viruses to facilitate gene transferability to cancer cells disseminated in the serosal
\end{abstract}

Correspondence to: Dr Robert M. Proczka, Second Department of General, Vascular and Oncological Surgery, Medical University of Warsaw, 19/25 Stepinska Str, 00-739 Warsaw, Poland

E-mail: ramjup@poczta.onet.pl

Key words: cancer gene therapy, gene transfer, cancer, effusion cavity, as well as the potential usefulness of these viruses as a new approach in cancer gene therapy.

\section{Introduction}

One of the most well-known and lethal gynecological malignancies is that of ovarian cancer. The molecular pathology of ovarian cancer is not well understood and various pathways of development are currently under investigation. p53 as well as $B R C A 1$ and BRCA2 gene (proteins) dysfunctions are broadly taken into consideration $(1,2)$. It is accepted that ovarian cancer as well as other malignancies begin growth in the primary site of transformation and then spread to the pelvic and abdominal cavities before metastasizing to distant organs. Peritoneal dissemination of cancer cells is characteristic of advanced stages of ovarian, breast and lung cancers, and is associated with poor patient survival (2). The presence of cancer cells in effusions complicates treatment protocols. Cell eradication involving the serosal cavities by a standard oncological approach is limited. One of the alternative therapeutic options available is cancer gene therapy.

Gene therapy represents a novel method of therapy of various diseases based on the introduction of genetic material in cells in order to correct their biological functions. The first gene therapy clinical trial was conducted by Blaese and coworkers in 1990 (3). However, many other studies are ongoing (4). Due to promising reports already published, the focus has been on cancer gene therapy trials (4). Nevertheless, it is obvious that future trials require new experiments to be conducted with a view to evaluating the mechanisms of cellular gene uptake. Currently, genes encoding therapeutic factors can be delivered into cells by viral and non-viral methods. The viral vectors are used worldwide in gene therapy clinical trials because of their efficiency of gene introduction in human cells (4). Among them, retrovirus- and adenovirus-derived genetic constructs are mainly used in the clinic (4). Wellknown advantages of retroviral vectors include the ability of these vectors to integrate into host cell genomes and to show a long-term expression of transgenes. A serious limitation, however, involves the possibility of inducing oncogenesis fol- 
lowing insertional mutagenesis $(5,6)$. A significant advantage of adenoviral constructs is their ability to infect both dividing and non-dividing cells, while an important disadvantage is the immune response against recombinant viruses (6).

Improving the biosafety and infection efficiency of viral vectors has been emphasized (6). Initial clinical applications used adeno-associated virus-derived vectors (rAAV) $(4,7)$. Approximately 50 rAAV-based gene therapy clinical trials have been reported worldwide (4). AAV belong to the Parvoviridae family, genus Dependovirus and represent small, $\sim 20 \mathrm{~nm}$ in diameter, icosahedral, non-enveloped particles containing single-stranded 4.7-kb DNA genomes (7). AAV were discovered in the 1960s as small adenovirus subunits or adenovirus-contaminating viruses (8). Finally, it was established that AAV represent small, defective, DNA-containing viruses which require a helper adenovirus or herpesvirus to replicate in host cells $(7,9)$. Significant advantages of AAV as genetic vehicles include the ability to infect a broad range of dividing and non-dividing cells and the lack of pathogenicity in humans. Additionally, AAV can integrate into a defined region of the human genome, and the observed transgene expression occurs over an extensive period of time $(7,9)$. Recombinant AAV vectors have been shown to infect normal tissues such as liver, muscle, skin, the nervous system and cancer cells $(4,7,10)$. The ability of rAAV vectors to introduce therapeutic genes to cancer cells disseminated into the peritoneal cavity has also been reported (11-13).

In this study, we considered viral vectors to be vehicles for gene transfer to cells disseminated in the serosal cavities. We showed a utility of recombinant adeno-associated virus vector serotype 2 (rAAV2) for the intraperitoneal administration of genes to cancer cells. rAAV2 vectors encoding reporter genes, green fluorescent protein $(G F P)$ and $\beta$-galactosidase $(L a c Z)$ were also investigated. The aim of the experiments was to evaluate the infection efficiency of L1 cancer cells by rAAV serotype 2 vectors in mouse peritoneal cavity. The results appear to be directly useful for the planning of cancer gene therapy clinical trials for ovarian cancer.

\section{Materials and methods}

\section{Production of recombinant adeno-associated virus sero-} type 2

Packaging cell culture. The AAV-293 cell line (Stratagene) was used for the production of recombinant infectious AAV particles. AAV2 was the only serotype produced and used in the studies. The AAV-293 cell line represents stable transformed by adenovirus type 5 DNA human embryonic kidney cells that have an adenovirus E1 gene required for rAAV production in vitro. For rAAV production, AAV-293 cells were maintained in Dulbecco's modified Eagle's medium (DMEM), supplemented with $10 \%$ fetal bovine serum (FBS), $4 \mathrm{mM}$ L-glutamine and $4.5 \mathrm{~g} / 1$ glucose at $37^{\circ} \mathrm{C}$ in a humidified atmosphere of $5 \% \mathrm{CO}_{2}$. The cells were cultured to a confluence of $60-70 \%$ and then the triple co-transfection procedure with AAV plasmids was performed.

Plasmid constructs. The expression plasmid vectors $\mathrm{pRC}$, pHelper, pGFP and pLacZ (Stratagene) were used in the experiments. The constructs contain AAV and adenovirus genes that are required for the production of infectious AAV particles. The pRC vector supplies rep and cap genes encoding AAV replication and capsid proteins, respectively. The pHelper vector contains the adenovirus E2A, E4, VA genes, and the pGFP or pLacZ vectors contain the $G F P$ and $\beta$-GAL reporter genes, respectively. The reporter vectors represent the ITRcontaining plasmids with $c m v$ promoters. The AAV plasmid vectors were amplified in transformed bacteria Escherichia coli and isolated by an alkaline lysis method using Endofree chromatographic columns (Qiagen). The quality of the isolated plasmids was confirmed spectrophotometrically and by restriction digestion mapping.

rAAV isolation and purification. rAAV infectious particles (rAAV vectors) were produced in a helper-free system, without using a helper adenovirus or herpesvirus for productive infection. The AAV-293 packaging cells were triple transient co-transfected with rAAV plasmid vectors pRC, pHelper and pGFP/pLacZ at a molar ratio of 1:1:1 using $25 \mathrm{kDa}$ polyethylenimine (PEI $25 \mathrm{kDa}$; Sigma) cationic polymer as a DNA carrier agent. The transfected cells were harvested after 48-72 h. To release rAAV virions, the transfected cells were lysed by the freeze-thaw method. The isolation and purification procedure was performed according to the Zolotukhin et al protocol (14). Briefly, after the temperature-dependent cell lysis, the crude lysate (virus-containing supernatant) was purified by iodixanol gradient ultracentrifugation, followed by heparin agarose affinity chromatography (Sigma). The rAAV crude lysate was placed into an iodixanol gradient column (comprising 15, 25, 40 and $60 \%$ fractions) and ultracentrifugated $\left(85 \mathrm{~min}, 18^{\circ} \mathrm{C}\right.$, $255,000 \mathrm{x} \mathrm{g}$ ). Immediately after centrifugation, the fraction between 40 and $60 \%$ was transferred into the heparin agarose column (Sigma). The purification was performed at room temperature conditions. The eluted primary virus stock was concentrated by centrifugation through the $100 \mathrm{MVCO}$ filter columns (Millipore) and stored at $-80^{\circ} \mathrm{C}$ for further studies.

$r A A V$ quantitative analysis. The number of genome copies of rAAV particles in obtained vector stocks (genome copies per ml) was determined by quantitative real-time PCR (SYBR ${ }^{\circledR}$ Green PCR Master Mix, Applied Biosystems). Assessment was performed using primers within the $c m v$ promoter sequence (forward: CACCAAAATCAACGGGACTT and reverse: GAGGTCAAAACAGCGTGGAT; 156-bp product; $\mathrm{T}_{\mathrm{m}}=50^{\circ} \mathrm{C}$ ). The $\mathrm{qPCR}$ curve of each AAV stock sample was related to the standard curve prepared using a 6 log spanning serial dilution of pLacZ plasmid construct containing one $\mathrm{cmv}$ promoter sequence per molecule. Each dilution step was measured in duplicate per run. The standard curve was calculated by the ABI Prism 7000 SDS software (Version 1.1; Applied Biosystems) by regression of the crossing points of the PCR curves from the dilution series of the vector plasmid. Melting curve analysis and agarose gel electrophoresis were performed, demonstrating specificity of the qPCR products.

Intraperitoneal infection of L1 cancer cells. The L1 cancer cells (mouse fibrosarcoma) were grown in vitro to a confluence of $70-80 \%$ in standard cell culture conditions. The cells were maintained in DMEM, supplemented with $10 \% \mathrm{FBS}$ at $37^{\circ} \mathrm{C}$ in a humidified atmosphere of $5 \% \mathrm{CO}_{2}$. The cells were then harvested and intraperitoneally (i.p.) injected to $\mathrm{Balb} / \mathrm{c}$ mice at a dose of $2-3 \times 10^{5}$ cells per mouse. Ten days later, the mice were i.p. injected with the rAAV preparations at different doses 


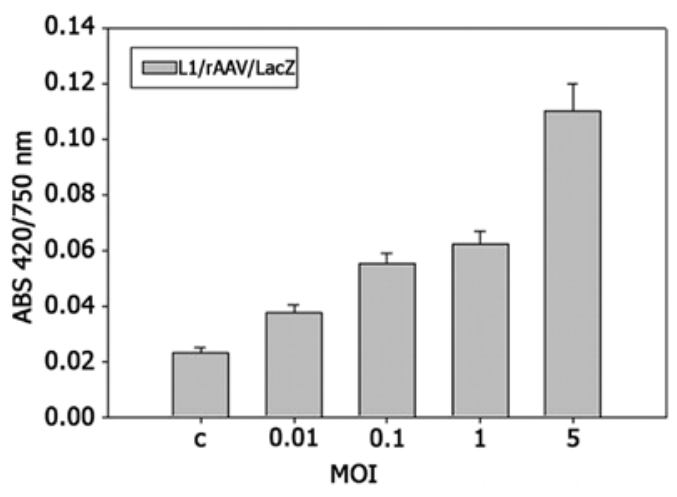

Figure 1. Intraperitoneal infection of L1 cancer cells by rAAV/LacZ vectors The cells were i.p. infected for three days at a MOI of $0.01,0.1,1$ and 5.

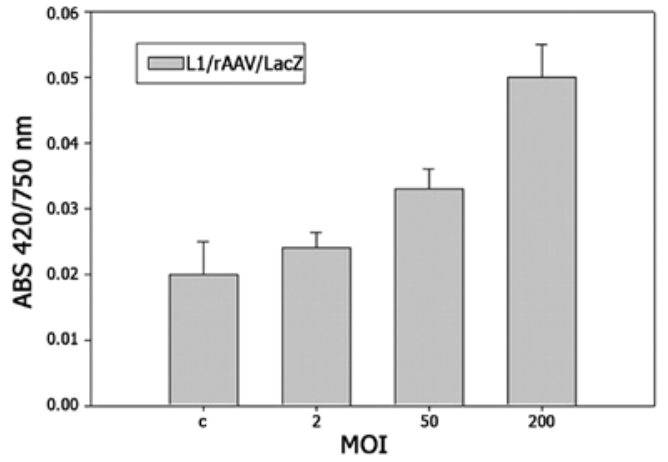

Figure 2. Intraperitoneal infection of L1 cancer cells by rAAV/LacZ vectors The cells were i.p. infected for three days at a MOI of 2, 50 and 200.

$\left(1.2 \times 10^{5}-4.2 \times 10^{6} \mathrm{gc} / \mathrm{ml}\right.$; MOI of $\left.0.01-200\right)$. After three days, the mice were sacrified and the intraperitoneal effusions containing infected cancer L1 cells were taken for further studies. The presence of virus DNA sequences as well as the expression of reporter genes in the studied cells were evaluated. The total DNA from the infected cells was isolated using the Qiamp mini kit (Qiagen) according to the manufacturer's protocol. The amplification of virus sequences was performed by a standard PCR method using specific starters (GAPDH: GAGTACGTCGTGGAGTCCACTGGCGTC3' and 5'CTTGA TGTCATCATATTTGGCAGGTTT3'; GFP: 5'AGCTGGAC GGCGACGTAAAC3' and 5'GTCGGCCATGATATAGACGT3'; LacZ: 5'CATCTGCTGCACGCGGAAGAA3' and 5'TACATG GTTGCTTTGACGT).

Reporter gene expression. To determine the intraperitoneal infection efficiency of cancer cells with a $\mathrm{rAAV} / \mathrm{LacZ}$ vector the $\beta$-galactosidase test was performed. The $\beta$-galactosidase enzyme assay was used to study $\beta$-galactosidase activity in L1/ rAAV/LacZ cell lysates prepared from L1 cells i.p. infected with viral particles. The test was performed according to the Promega $\beta$-galactosidase Assay System protocol with ONPG (O-nitrophenyl- $\beta$-D-galactopyranoside; Sigma) as a substrate. $\beta$-galactosidase derived from rAAV/LacZ-infected L1 cells hydrolyzes the colorless ONPG substrate to yellow o-nitrophenol, the content of which is defined with a spectrophotometer at an absorbance of $420 \mathrm{~nm}$. Additionally, the total protein
Table I. Number of GFP-positive L1 cells found in mouse intraperitoneal effusion. ${ }^{\mathrm{a}}$

\begin{tabular}{|c|c|c|c|c|c|}
\hline \multicolumn{6}{|c|}{ Multiplicity of infection (MOI) } \\
\hline $\mathrm{C}$ & 0.001 & 1 & 2 & 3 & 10 \\
\hline \multicolumn{6}{|c|}{ No. of L1/rAAV/GFP cells } \\
\hline $\begin{array}{l}0.0 \\
\mathrm{n}=3\end{array}$ & $\begin{array}{l}0.0 \\
n=3\end{array}$ & $\begin{array}{c}0.0 \\
\mathrm{n}=4\end{array}$ & $\begin{array}{c}3.5 \pm 1.7 \\
\mathrm{n}=3\end{array}$ & $\begin{array}{c}9.0 \pm 5.7 \\
n=3\end{array}$ & $\begin{array}{c}17 \pm 2.3 \\
\mathrm{n}=5\end{array}$ \\
\hline
\end{tabular}

a $\mathrm{L} 1$ cancer cells were i.p. infected with $\mathrm{rAAV} / \mathrm{GFP}$ vectors at a dose calculated as a MOI $(0.001,1,2,3$ and 10$)$. L1/rAAV/GFP cells were counted under an inverted fluorescence microscope. Results represent the mean \pm SD. C, control.

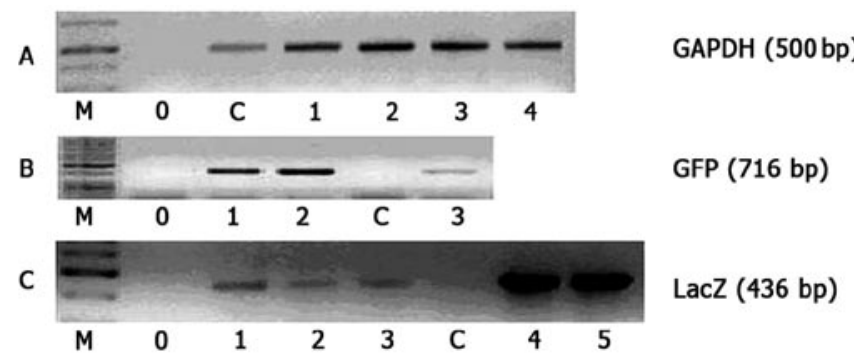

Figure 3. PCR analysis of the rAAV vector sequence presence in the i.p. L1-infected cells. The analysis was performed by standard PCR using total DNA isolated from the i.p. L1-infected cells. (A) Amplification of the 500-bp fragment of GAPDH (marker gene); lanes 1 and 2, L1/rAAV/GFP (MOI=3); lanes 3 and 4, L1/rAAV/LacZ (MOI=50). (B) Amplification of the 716-bp fragment of the GFP gene: lanes 1 and 2, L1/rAAV/GFP (MOI=3); lane 3, positive control (plasmid pEGFP). (C) Amplification of the 436-bp fragment of the $\beta$-galactosidase gene; lanes 1,2 and 3, L1/rAAV/LacZ (MOI=5); lanes 4 and 5, L1/rAAV/LacZ (MOI=50); 0, negative control (without DNA). $\mathrm{C}$, control DNA from non-infected cells.

content was determined by the Lowry method (absorbance was read at $750 \mathrm{~nm}$ ). The relative $\beta$-galactosidase activity was calibrated as the ratio of $\beta$-GAL $420 \mathrm{~nm}$ absorbance to the total protein $750 \mathrm{~nm}$ absorbance (ABS 420/750 nm). The GFPpositive L1 cancer cells were also evaluated. The i.p.-infected L1 cancer cells with rAAV/GFP vectors were observed and counted under an inverted fluorescence microscope (Olympus IX51).

\section{Results}

Recombinant adeno-associated viral vectors (rAAV2) were produced in adenovirus-free conditions by a transient transfection of AAV-293 packaging cells with AAV plasmid constructs encoding essential viral genes. Based on a determination of virus genome copies performed by quantitative real-time PCR, it was estimated that produced virus stocks contain 0.3$0.7 \times 10^{8} \mathrm{gc} / \mathrm{ml}$. Experiments evaluating the infectious activity of obtained recombinant adeno-associated virus vectors encoding reporter genes (GFP and $\beta$-GAL) were performed in vivo on Balb/c mice i.p. injected with L1 cancer cells. The cells were i.p. infected for three days with rAAV vectors encoding reporter genes. rAAV preparations were injected directly into the mouse peritoneal cavity at a multiplicity of infection (MOI) 
ranging from 0.01 to 200 . The transgene expression was tested on L1-infected cells involving the peritoneal effusions.

Figs. 1 and 2 show that recombinant AAV effectively infect L1 cells disseminated in the peritoneal cavity. The cells revealed an ability to uptake the rAAV vectors and express the reporter $\beta$-GAL gene. Figs. 1 and 2 show that the infection efficiency depends on the virus dose used in the experiments. The infection, calculated as $\beta$-galactosidase activity vs. total protein content (ABS420/750) increases with a MOI ratio. The highest expression of the LacZ reporter gene was observed at a MOI of 50 and 200. Studies performed with $\mathrm{rAAV} / \mathrm{GFP}$ vectors also revealed that the i.p. infection is closely dependent on the virus particle number injected into the peritoneal cavity (Table I). As shown in Fig. 3, L1 cells infected with rAAV displayed presence of the virus sequence in their genome. Amplification of the fragment of the $\operatorname{rAAV} \beta-G A L$ and $G F P$ genes by PCR confirmed the intraperitoneal infection of L1 cells by recombinant adeno-associated viruses.

\section{Discussion}

The presence of cancer cells in the effusion fluid involving the serosal cavities is a frequent clinical finding. As described, cancer cells disseminated into the peritoneal, pleural or pericardial cavities have a unique biology. The expression pattern of various cell proteins may vary between cancer types at the primary site (solid tumors) and their effusion counterparts $(2,15)$. The accumulation of cancer cells in the effusions has clinical prognostic importance and directly indicates efficiency of the treatment of serious malignancies. It is known that the eradication of cancer cells and effusion fluids from serosal cavities is very difficult because of surgical limitations. Additionally, the usefulness of chemotherapy is poor, since cancer cells in effusions often reveal a resistance to apoptosis (15). Gene therapy and gene preparations represent a novel therapeutic approach in contemporary medicine. The focus lies on engineering gene transfer vectors that effectively deliver to cancers genes, thereby encoding therapeutic proteins such as antiangiogenic (soluble receptors of vascular endothelial growth factors), proapoptotic (caspases, tissue inhibitors of matrix metalloproteinases) or immunomodulating (interleukins) (16). Cancer gene therapy is currently one of the most advanced gene therapy strategies found in the clinic (4). Preliminary outcomes underline the clinical significance of recombinant virus vectors for cancer cell infection (4). Thus, rAAV vectors are one of the more promising vehicles for gene delivery. Experimental observations confirm their biosafety and infection efficiency $(7,10)$. rAAV is able to transfer genes to cancer cells involving the serosal cavities as previously described (11-13). In this study, we showed the ability of rAAV2 vector encoding reporter genes, GFP and $\beta$-GAL, to infect fibrosarcoma cells disseminated into the mouse peritoneal cavity. We observed that rAAV2 was ingested by L1 cancer cells. The efficiency of infection was correlated to a MOI ratio. These experiments showed that rAAV has delivered the gene of interest to cancer cells in the peritoneal cavity. Notably, no toxicity was observed (data not shown). The utility of recombinant virus vectors for gene transfer to cells involving the serosal cavities is tentatively reported by others. Studies published by Isayeva et al revealed that recombinant AAV vectors encoding antiangiogenic factors (angiostatin and endostatin) may be useful for the treatment of intraperitoneal ovarian cancer dissemination $(11,12)$. The possibility of preventing ovarian cancer metastasis through intraperitoneal rAAV-mediated gene transfer was also described by Li et al (13). The authors underlined the benefit of the rAAV-dependent long-term expression of the $\mathrm{nm} 23 \mathrm{HI}$ gene in vivo.

Gene therapy and gene-based preparations generate a new strategy for cancer treatment. Preliminary observations demonstrate the ability of recombinant adeno-associated viruses to facilitate therapeutic gene delivery to cancer cells disseminated in the serosal cavities. Our study illustrates that rAAV 2 vectors are useful for the intraperitoneal infection of cancer cells. The findings appear to be important for cancer gene therapy clinical trials for ovarian, breast or pancreas cancers.

\section{Acknowledgements}

This work was supported by a grant from the Polish Committee for Scientific Research (KBN 2P05E03328).

\section{References}

1. Reibenwein $\mathbf{J}$ and Krainer M: Targeting signaling pathways in ovarian cancer. Expert Opin Ther Targets 12: 353-365, 2008.

2. Cannistra SA: Cancer of ovary. N Engl J Med 351: 2519-2529, 2004.

3. Blaese RM, Culver KW, Miller AD, et al: T lymphocyte-directed gene therapy for ADA-SCID: initial trial results after 4 years. Science 270: 475-480, 1995.

4. www.wiley.co.uk

5. Porteus MH, Connelly JP and Pruett SM: A look to future directions in gene therapy research for monogenic diseases. PLoS Genet 2: 1285-1292, 2006.

6. Raty JK, Lesch HP, Wirth T and Ylä-Herttuala S: Improving safety of gene therapy. Curr Drug Saf 3: 46-53, 2008.

7. Le Bec C and Douar A: Gene therapy progress and prospectsvectorology: design and production of expression cassettes in AAV vectors. Gene Ther 13: 805-813, 2006.

8. Atchison RW, Casto BC and Hammon WM: Adenovirusassociated defective virus particles. Science 149: 754-756, 1965.

9. Małecki M, Woźniak A and Janik P: Wirusy związane z adenowirusami (AAV). Postępy Biochem 54: 57-63, 2008.

10. Flotte TR: Gene therapy progress and prospects: recombinant adeno-associated virus (rAAV) vectors. Gene Ther 11: 805-810, 2004.

11. Isayeva $\mathrm{T}$, Ren $\mathrm{C}$ and Ponnazhagan S: Recombinant adenoassociated virus 2-mediated antiangiogenic prevention in a mouse model of intraperitoneal ovarian cancer. Clin Cancer Res 11: 1342-1347, 2005.

12. Isayeva T, Ren $C$ and Ponnazhagan $S$ : Intraperitoneal gene therapy by rAAV provides long-term survival against epithelial ovarian cancer independently of survivin pathway. Gene Ther 14: 138-146, 2007.

13. Li J, Zhou J, Chen G, et al: Inhibition of ovarian cancer metastasis by adeno-associated virus-mediated gene transfer of $\mathrm{nm} 23 \mathrm{H} 1$ in an orthotopic implantation model. Cancer Gene Ther 13: 266-272, 2006.

14. Zolotukhin S, Byrne BJ, Mason E, et al: Recombinant adenoassociated virus purification using novel methods improves infectious titer and yield. Gene Ther 6: 973-985, 1999.

15. Davidson B: Biological characteristics of cancers involving the serosal cavities. Crit Rev Oncog 13: 189-227, 2007.

16. Malecki M, Kolsut P and Proczka P: Angiogenic and antiangiogenic gene therapy. Gene Ther 12: 159-169, 2005. 